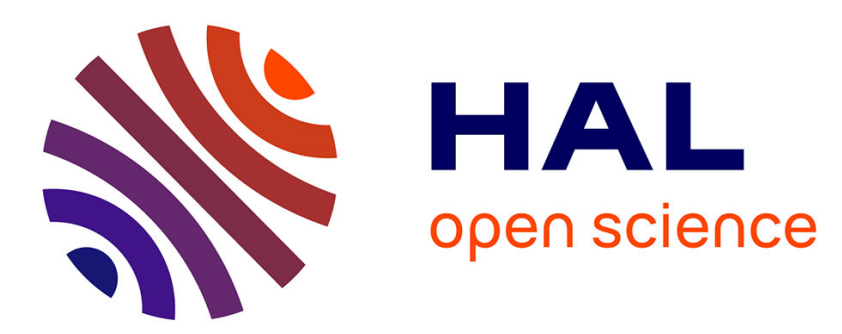

\title{
Un nouvel Agraecia de Martinique et présence de Agraecia viridipennis en Guyane française (Orthoptera, Ensifera, Conocephalinae)
}

Sylvain Hugel

\section{- To cite this version:}

Sylvain Hugel. Un nouvel Agraecia de Martinique et présence de Agraecia viridipennis en Guyane française (Orthoptera, Ensifera, Conocephalinae). Bulletin de la Société Entomologique de France, 2009, 114 (2), pp.129-140. 10.3406/bsef.2009.2795 . hal-03431833

\section{HAL Id: hal-03431833 \\ https://hal.science/hal-03431833}

Submitted on 16 Nov 2021

HAL is a multi-disciplinary open access archive for the deposit and dissemination of scientific research documents, whether they are published or not. The documents may come from teaching and research institutions in France or abroad, or from public or private research centers.
L'archive ouverte pluridisciplinaire HAL, est destinée au dépôt et à la diffusion de documents scientifiques de niveau recherche, publiés ou non, émanant des établissements d'enseignement et de recherche français ou étrangers, des laboratoires publics ou privés. 


\section{Un nouvel Agraecia de Martinique et présence de Agraecia} viridipennis en Guyane française (Orthoptera, Ensifera, Conocephalinae)

\section{Sylvain Hugel}

\section{Résumé}

Une nouvelle espèce du genre Agraecia Serville, 1831, est décrite de Martinique : A. cesairei n. sp. La stridulation de cette espèce est décrite et des éléments de biologie sont donnés. Agraecia viridipennis Redtenbacher, 1891, est signalé pour la première fois de Guyane française. La série de spécimens collectés sur ce territoire permet d'établir la synonymie d"Agraecia malkini Piza, 1978, avec A. viridipennis, qui est redécrit. La probable synonymie d'A. pulchella Hebard, 1927, avec A. vittata Redtenbacher, 1891, est évoquée, et l'homogénéité du genre Agraecia est discutée.

\section{Abstract}

A new Agraecia from Martinique and record of Agraecia viridipennis in French Guyana (Orthoptera, Ensifera, Conocephalinae). A new species of Agraecia Serville, 1831, is described from Martinique : $H$. cesairei $n$. sp. The song of this new species is described and biological data are given. Agraecia viridipennis Redtenbacher, 1891, is recorded for the first time in French Guyana. The specimens collected there allow synonymising Agraecia malkini Piza, 1978, with A. viridipennis which is redescribed. The probable synonymy of A. pulchella Hebard, 1927, with A. vittata Redtenbacher, 1891 , is pointed out, and the homogeneity of Agraecia is discussed.

\section{Citer ce document / Cite this document :}

Hugel Sylvain. Un nouvel Agraecia de Martinique et présence de Agraecia viridipennis en Guyane française (Orthoptera, Ensifera, Conocephalinae). In: Bulletin de la Société entomologique de France, volume 114 (2),2009. pp. 129-140; https://www.persee.fr/doc/bsef_0037-928x_2009_num_114_2_2795

\section{Ressources associées :}

Agraecia viridipennis

Fichier pdf généré le 08/10/2019 


\title{
Un nouvel Agraecia de Martinique et présence de Agraecia viridipennis en Guyane française (Orthoptera, Ensifera, Conocephalinae)
}

\author{
par Sylvain HUGEL \\ UPR-3212 CNRS, Université de Strasbourg, 21 rue Descartes, F - 67084 Strasbourg cedex \\ $<$ hugel@neurochem.u-strasbg.fr>
}

Résumé. - Une nouvelle espèce du genre Agraecia Serville, 1831, est décrite de Martinique : A. cesairei n. sp. La stridulation de cette espèce est décrite et des éléments de biologie sont donnés. Agraecia viridipennis Redtenbacher, 1891, est signalé pour la première fois de Guyane française. La série de spécimens collectés sur ce territoire permet d'établir la synonymie d'Agraecia malkini Piza, 1978, avec A. viridipennis, qui est redécrit. La probable synonymie d'A. pulchella Hebard, 1927, avec A. vittata Redtenbacher, 1891, est évoquée, et l'homogénéité du genre Agraecia est discutée.

Summary. - A new Agraecia from Martinique and record of Agraecia viridipennis in French Guyana (Orthoptera, Ensifera, Conocephalinae). A new species of Agraecia Serville, 1831, is described from Martinique: H. cesairei n. sp. The song of this new species is described and biological data are given. Agraecia viridipennis Redtenbacher, 1891, is recorded for the first time in French Guyana. The specimens collected there allow synonymising Agraecia malkini Piza, 1978, with A. viridipennis which is redescribed. The probable synonymy of $A$. pulchella Hebard, 1927, with $A$. vittata Redtenbacher, 1891, is pointed out, and the homogeneity of Agraecia is discussed.

Key words. - Agraeciini, Caribbean, West Indies, Lesser Antilles, Guyana, endemism, cone-head katydid.

New synonymy : Agraecia viridipennis Redtenbacher, 1891 = Agraecia malkini Piza, 1978, n. syn.

Le genre Agraecia a été érigé par AUDINET-SERVILLE (1831) et inclut actuellement dixsept espèces dont treize appartiennent à la faune néotropicale et quatre à la faune afrotropicale et orientale. Ces dernières espèces non néotropicales appartiennent très probablement à des genres distincts et ne sont pas traités dans les diagnoses du présent article.

Une dizaine de missions dans les Petites Antilles m'ont permis de collecter de très nombreuses espèces d'Orthoptères parmi lesquelles plusieurs se sont avérées nouvelles pour la science (Hugel, in prep.), dont une nouvelle Agraecia qui semble endémique de Martinique. Une seule espèce d'Agraeciini est actuellement connue des Antilles : Erechthis gundlachi Bolívar, 1888, de Cuba et Porto-Rico ; aucune n'est connue des Petites Antilles. Par les tympans des tibias antérieurs non largement ouverts; les fémurs armés ventalement; la face sans carènes ; le prosternum armé ; le haut du fastigium du vertex sans épine ; les élytres bien développés et non tronqués à l'apex; les scapes sans tubercule ou épine interne; la métazone du pronotum pas particulièrement développée postérieurement, l'espèce décrite dans le présent article appartient clairement au genre Agraecia.

$\mathrm{Au}$ cours de plusieurs voyages en Guyane française, j'ai collecté dans une même localité quelques spécimens d'une espèce d'Agraecia. Les femelles correspondent à l'holotype femelle de A. viridipennis Redtenbacher, 1891, et le mâle correspond à l'holotype mâle de A. malkini Piza, 1978.

Dans la première partie du présent article, Agraecia cesairei n. sp. est décrite, sa stridulation est détaillée et des caractéristiques de sa biologie sont données; dans la seconde partie du présent article, Agraecia viridipennis Redtenbacher, 1891, est signalée de Guyane française, la synonymie de A. malkini avec A. viridipennis est établie et un complément de description de cette espèce est donné. 


\section{Agraecia cesairei n. sp.}

HolotyPE: ${ }^{\lambda}$, Antilles, Martinique, Fonds-Saint-Denis, plateau Boucher, sentier du Carbet, 650 m alt., $61^{\circ} 06^{\prime} 00^{\prime \prime} \mathrm{O}-14^{\circ} 43^{\prime} 09^{\prime \prime} \mathrm{N}, 11 . \mathrm{VIII} .2008, \mathrm{n}^{\circ}$ spn et enregistrement Mart 2008081 , S. Hugel, MNHN.

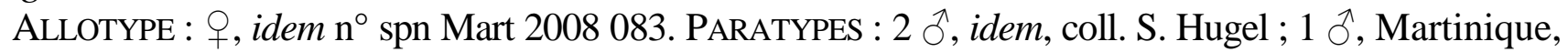
Fonds-Saint-Denis, Arborétum de l'Alma, $500 \mathrm{~m}$ alt., $61^{\circ} 05^{\prime} 38^{\prime \prime O}-14^{\circ} 42^{\prime} 18^{\prime \prime N}$, 19.IX.2006, M. Boucherit \& J. Weber leg., coll. S. Hugel ; 1 §ิ, idem, Musée zoologique de Strasbourg ; 1 q, idem, 3.VIII.2008, S. Hugel, S. Hugel coll. ; 1 +, Martinique, Fonds-Saint-Denis, départ nord-ouest de la Trace des Jésuites,

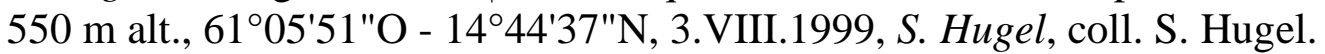

Diagnose. - Cette espèce nouvelle se distingue de toutes les autres Agraecia néotropicales par la combinaison des caractères suivants: face entièrement noire (hormis l'ocelle médian et le clypéus qui sont blancs; les autres espèces ont au plus une bande longitudinale noire comme A. nigrifrons Redtenbacher, 1891, et A. ornata Karny, 1907); face relativement étroite et allongée ; ocelle médian de petite taille, son centre au niveau de la base des scrobes antennaires (ocelle médian plus large qu'un œil chez A. viridipennis Redtenbacher, 1891); face lisse (avec des dépressions chez A. ornata); apex du fastigium du vertex en angle aigu émoussé (en éperon chez A. subulata Redtenbacher, 1891 ; en pointe chez A. maculata Redtenbacher, 1891, arrondi chez A. festae Griffini, 1896, et A. viridipennis) ; fastigium du vertex frontalement formant une crête étroite (large et aplati chez A. incognita Piza, 1970) ; élytres dépassant largement les genoux postérieurs (les atteignant à peine chez A. abbreviata Redtenbacher, 1891, et $A$. vittipes Redtenbacher, 1891, et les dépassant légèrement chez $A$. ornata); élytres $>35 \mathrm{~mm}(<25 \mathrm{~mm}$ chez $A$. festae $)$ avec taches sombres irrégulières aux limites peu définies (base des élytres avec des taches noires irrégulières bien nettes chez $A$. viridipennis); fémurs antérieurs avec des épines ventrales postérieures (absentes chez $A$. viridipennis) ; fémurs postérieurs élancés, plus de 6 fois aussi longs que larges (fémurs postérieurs trapus, environ 4 à 4,5 fois plus longs que large chez A. vittata Redtenbacher, 1891 et A. pulchella Hebard, 1927); miroir de l'élytre gauche du mâle membraneux/translucide (miroir gauche opaque avec de nombreuses veines chez A. viridipennis); plaque sous-génitale du mâle avec une émargination profonde (émargination superficielle chez A. incognita); cerques du mâle prolongées par une très longue protrusion horizontale finissant en pointe vers le haut (cerques du mâle sans très longue protrusion chez A. dorsalis Karny, 1907, A. punctata, $A$. subulata et $A$. vittipes) ; plaque sous-génitale de la femelle avec une large émargination (émargination étroite en $\mathrm{V}$ chez $A$. incognita ; émargination très superficielle chez $A$. viridipennis) ; oviscapte égal aux deux tiers de la longueur du fémur postérieur (à peine plus court chez $A$. vittipes et $A$. ornata); oviscapte légèrement et régulièrement incurvé vers le haut (brusquement incurvé au milieu chez A. pulchella; excurvé chez A. incognita), longueur supérieure à $15 \mathrm{~mm}$ ( $9 \mathrm{~mm}$ chez $A$. festae $)$.

Description. - Outre les caractéristiques générales du genre (KARNY, 1912).

Couleur. Adultes : coloration générale brune; face, scape, genoux, apex des tibias, épines des fémurs noirs ; clypéus, labrum, palpes, ocelle médian, ocelles latéraux, apex du fastigium du vertex blancs ; élytres bruns avec taches sombres indistinctes; abdomen jaune sur les spécimens vivants (fig. 1 et 2). Jeunes : à l'éclosion et premiers stades verts; chez les derniers stades coloration générale beige clair, occiput, pronotum, tergites abdominaux avec lignes longitudinales brunes.

Tête. Face lisse. Fastigium du vertex atteignant le milieu du scape (vue frontale, fig. 2), dépassant à peine les scrobes antennaires (vue dorsale, fig. 3) ; apex du fastigium en angle aigu émoussé (fig. 4); base du fastigium du vertex étroite, concave, largement dépassée par les scrobes antennaires (vue semi latérale, scapes relevés). Ocelle médian bien distinct (fig. 2), centre situé au niveau de la base des scrobes, diamètre distinctement inférieur au diamètre d'un œil. Occiput avec des sillons longitudinaux et transverses irréguliers. Yeux petits, peu saillants, légèrement allongés en vue dorsale. Antennes $>80 \mathrm{~mm}$. 
Thorax. Pronotum : lisse, brillant, avec de nombreuses dépressions ; bord antérieur légèrement concave (fig. 3), bord postérieur presque droit, légèrement convexe ; sillon antérieur peu distinct sur le disque, sillon postérieur bien distinct; sillon sagittal distinct tout le long du disque ; bord ventral du lobe latéral légèrement concave ; sinus huméral distinctement concave (fig. 1). Epines du prosternum longues, légèrement incurvées vers l'avant.

Elytres. Dépassant nettement les genoux postérieurs.

Pattes. Tibia antérieur : arrondi dorsalement, aplati ventralement, avec 1 éperon apical ventral de part et d'autre, et ventralement 7-8 éperons subapicaux internes (7 chez l'holotype et l'allotype) et 6-8 éperons subapicaux externes (7-8 chez l'holotype et l'allotype); renflement tympanique à peine marqué, orifices tympaniques ouverts vers l'avant, plus étroits que l'espace qui les sépare. Fémur antérieur: arrondi dorsalement, avec deux carènes longitudinales ventrales, avec 5-7 (6 chez l'holotype et 5 chez l'allotype) épines ventrales internes, avec 3-5 épines ventrales externes (4 chez l'holotype et 3-4 chez l'allotype) ; lobe géniculaire interne pointant légèrement ventralement ; lobe géniculaire externe plus arrondi. Epine du coxa antérieur étroite en vue dorsale. Tibia médian : arrondi dorsalement, aplati ventralement à l'apex ; avec un éperon apical ventral de part et d'autre ; avec ventralement 8-11 éperons subapicaux antérieurs (11 chez l'holotype et 8-10 chez l'allotype) et 5-8 éperons subapicaux postérieurs ( 7 chez l'holotype et 6 chez l'allotype). Fémur médian : arrondi dorsalement ; avec une carène ventrale antérieure et une carène ventrale postérieure ; avec 6-10 épines ventrales antérieures (6-7 chez l'holotype et 6-8 chez l'allotype); sans épines ventrales postérieures (sauf sur un spécimen avec 1 petite épine) ; lobe géniculaire postérieur pointant légèrement ventralement; lobe géniculaire antérieur plus arrondi. Coxa médiane avec une petite épine distale dorsale antérieure et un tubercule distal ventral. Tibia postérieur : arrondi ventralement, apex aplati (deux carènes ventrales apparaissent à l'apex); aplati dorsalement; avec 2 éperons apicaux ventraux de part et d'autre et 1 éperon apical dorsal de part et d'autre ; avec 6-8 éperons subapicaux ventraux internes (7 chez l'holotype et 8 chez l'allotype); avec 10-12 éperons subapicaux ventraux externes (11 chez l'holotype et l'allotype); avec 15-16 épines dorsales internes (16 chez l'holotype et l'allotype), et 12-14 épines dorsales externes (14 chez l'holotype et 13 chez l'allotype). Fémur postérieur : arrondi dorsalement, avec deux carènes ventrales ; avec 1-2 épines ventrales internes ( 1 chez l'holotype ; 2 chez l'allotype) ; avec 7-9 épines ventrales externes (8 chez l'holotype ; 8-9 chez l'allotype) ; lobes géniculaires terminés par une épine de part et d'autre.

Dimorphisme sexuel. Hormis les caractères sexuels primaires, les deux sexes sont similaires.

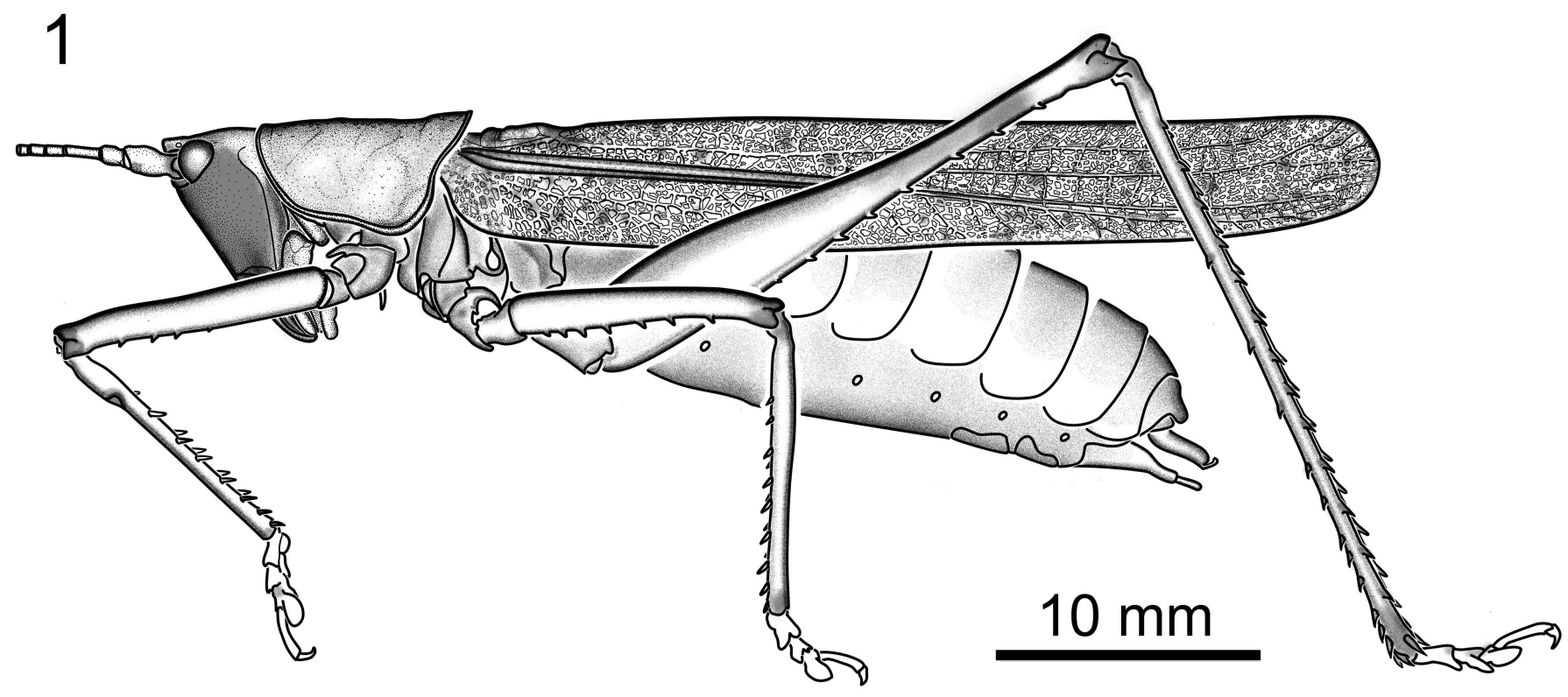

Fig. 1. - Agraecia cesairei n. sp., ô paratype.

Mâle. - Appareil stridulatoire. Elytre gauche (fig. 10 et 12) : crête stridulatoire en vue dorsale de $1.1 \mathrm{~mm}$ environ (vue dorsale) ; râpe avec 68-71 lames (holotype : 71) ; miroir translucide, membraneux. Elytre droit (fig. 11) : miroir au milieu aussi large que long (élytre fermé ; moyenne 1,0 ; min : 0,9 ; $\max : 1,1$; holotype 1.0) distalement au miroir, champ anal avec de nombreuses soies courtes spiniformes.

Aile. Base de l'aile avec une évagination molle dressée vers le haut (visible aile ouverte, principalement sur les spécimens frais ou en alcool ; fonction glandulaire ?). Terminalia. Plaque suranale 
légèrement échancrée ; cerques (fig. 5, 6 et 9) très allongés, base large avec de nombreuses soies ; base prolongée d'une longue protrusion horizontale étroite terminée par une dent dirigée vers le haut (fig. 5) ; certains spécimens avec un renflement postérieur externe de taille variable à la limite entre la base du cerque et la protrusion (fig. 6 et 9). Plaque sous-génitale (fig. 9) allongée, 1.1-1.6 fois aussi longue (sans les styli) que large à la base ; bord postérieur avec une échancrure en $\mathrm{V}, 0.22$ (min : 0.20 ; max : 0.24) fois aussi profonde que la longueur maximale de la plaque sous-génitale ; carènes latérales distinctes à l'apex, sub-parallèles ; styli bien distincts, allongés. Genitalia. Titillateurs (fig. 27) symétriques, plus hauts que large, arqués vers l'extérieur, accolés au milieu, légèrement sclérifiés, avec des tubercules dans la partie supérieure.

Femelle. - Terminalia: sternite précédant la plaque sous-génitale avec un petit renflement au milieu; plaque sous génitale (fig. 7 et 8) plus large que longue, avec deux carènes longitudinales médianes peu distinctes à la base ; émargination apicale large, carrée ; oviscapte droit à la base, légèrement incurvé, avec de minuscules denticulations peu distinctes sur les valves dorsales à partir du milieu, et à l'apex des valves ventrales; base des valves ventrales avec de part et d'autre un petit renflement indistinct dirigé vers l'extérieur (sans épine).

Stridulation. - Le chant d'appel de Agraecia cesairei $\mathrm{n}$. sp. est produit de nuit, par des mâles perchés à 1-5 mètres de haut sur des plantes de sous-bois. Un seul spécimen a été enregistré directement sur le terrain, mais la moitié des mâles collectés l'ont été au moyen d'un détecteur d'ultrasons. Le spécimen enregistré a été concomitamment filmé avec une caméra infrarouge pour s'assurer de l'origine de l'enregistrement. A $26^{\circ} \mathrm{C}$, le chant est composé de séquences irrégulièrement répétées toutes les 76,4 $\pm 124,7 \mathrm{~s} \mathrm{(min} \mathrm{:} \mathrm{3,0} \mathrm{;} \mathrm{max} \mathrm{;} \mathrm{363,7} \mathrm{;}$ $\mathrm{n}=9$ séquences). Chaque séquence comprend de 2 à 3 phrases $(2,3 \pm 0,5 ; \mathrm{n}=9$ séquences $)$ irrégulièrement espacées de $1188 \pm 652 \mathrm{~ms}(\min : 338 ; \max : 2351 ; \mathrm{n}=21$ phrases). Chaque phrase dure $249 \pm 33 \mathrm{~ms}$ (min : 189; $\max : 318 ; \mathrm{n}=21$ phrases) et comprend 6,5 $\pm 1,0(\min : 5 ; \max : 8)$ syllabes dont les premières sont plus courtes et isolées que les dernières. La première harmonique est à la fréquence de 19-22 kHz. Dans

Tableau I. - Mesures de Agraecia cesairei n. sp. (en mm).

\begin{tabular}{|c|c|c|c|c|c|c|c|c|c|c|c|}
\hline & \multirow{3}{*}{$\begin{array}{c}\text { Corps } \\
\mathrm{L}\end{array}$} & \multirow{2}{*}{\multicolumn{2}{|c|}{ Tête (face) }} & \multirow{2}{*}{\multicolumn{3}{|c|}{ Prn1 }} & \multirow{2}{*}{\multicolumn{2}{|c|}{ Elytre }} & \multicolumn{3}{|c|}{ Tibias } \\
\hline & & & & & & & & & \multirow{2}{*}{$\frac{\mathrm{T} 1}{\mathrm{~L}}$} & \multirow{2}{*}{$\frac{\mathrm{T} 2}{\mathrm{~L}}$} & \multirow{2}{*}{$\frac{\mathrm{T} 3}{\mathrm{~L}}$} \\
\hline & & $1 \max ^{1}$ & $1 \min ^{2}$ & $\mathrm{~L}$ & 1 & $\mathrm{~h}$ & $\mathrm{~L}$ & $1^{3}$ & & & \\
\hline ô holotype & 39,0 & 5,7 & 4,4 & 9,0 & 5,6 & 4,0 & 40,5 & 5,9 & 11,1 & 11,9 & 27,0 \\
\hline$\lambda \quad$ moyenne & 37,4 & 5,4 & 4,2 & 8,6 & 5,7 & 4,0 & 38,1 & 5,2 & 10,7 & 11,2 & 25,1 \\
\hline$\underset{(n-5)}{\text { minimum }}$ & 33,5 & 5,2 & 4,1 & 8,3 & 5,4 & 3,9 & 35,4 & 4,5 & 10,5 & 10,9 & 24,2 \\
\hline (II=J) maximum & 40,6 & 5,7 & 4,4 & 9,0 & 6,4 & 4,0 & 40,5 & 5,9 & 11,1 & 11,9 & 27,0 \\
\hline o allotype & 33,0 & 5,7 & 4,3 & 9,0 & 6,5 & 3,9 & 38,4 & 6,0 & 11,6 & 12,2 & 25,7 \\
\hline moyenne & 34,6 & 5,7 & 4,3 & 8,8 & 6,3 & 4,0 & 39,5 & 5,7 & 11,5 & 12,0 & 25,9 \\
\hline$\stackrel{+}{+}$ minimum & 33,0 & 5,7 & 4,3 & 8,7 & 6,1 & 3,9 & 38,4 & 5,1 & 11,3 & 11,9 & 25,7 \\
\hline maximum & 36,4 & 5,7 & 4,4 & 9,0 & 6,5 & 4,1 & 40,9 & 6,0 & 11,6 & 12,2 & 26,1 \\
\hline
\end{tabular}

\begin{tabular}{|c|c|c|c|c|c|c|c|c|c|c|c|}
\hline & \multicolumn{4}{|c|}{ Fémurs } & \multirow{2}{*}{\multicolumn{4}{|c|}{ Plaque sous-génitale }} & \multirow{3}{*}{$\begin{array}{c}\text { Styli } \\
\text { L }\end{array}$} & \multirow{2}{*}{\multicolumn{2}{|c|}{ Oviscapte }} \\
\hline & \multirow{2}{*}{$\frac{\mathrm{F} 1}{\mathrm{~L}}$} & \multirow{2}{*}{$\frac{\mathrm{F} 2}{\mathrm{~L}}$} & \multicolumn{2}{|c|}{$\mathrm{F} 3$} & & & & & & & \\
\hline & & & $\mathrm{L}$ & $1 \max$ & $\mathrm{L} \max$ & 1 base & 1 apex & L ém ${ }^{4}$ & & $\mathrm{~L}$ & $1^{3}$ \\
\hline$\hat{~ h o l o t y p e}$ & 11 & 10,4 & 25,0 & 3,8 & 4,1 & 2,7 & 1,9 & 0,9 & 1,1 & & \\
\hline moyenne & 10,5 & 10,3 & 24,3 & 3,6 & 3,7 & 2,7 & 1,7 & 0,8 & 1,0 & & \\
\hline minimum & 10,3 & 10,0 & 23,6 & 3,4 & 3,5 & 2,2 & 1,6 & 0,7 & 0,9 & & \\
\hline$(n=5) \frac{\text { maximum }}{n}$ & 11,0 & 10,4 & 25,0 & 3,8 & 4,1 & 3,7 & 1,9 & 0,9 & 1,1 & & \\
\hline q allotype & 11,0 & 10,7 & 25,0 & 3,6 & 2,4 & 3,4 & 1,4 & 0,6 & & 16,2 & 4,5 \\
\hline o moyenne & 11,0 & 10,8 & 25,4 & 3,6 & 2,5 & 3,5 & 1,3 & 0,7 & & 16,4 & 4,7 \\
\hline$(n=3)$ minimum & 10,9 & 10,7 & 25,0 & 3,6 & 2,4 & 2,9 & 1,1 & $\overline{0,6}$ & & 16,0 & $\overline{4,5}$ \\
\hline maximum & 11,1 & 11,0 & 26,2 & 3,7 & 2,6 & 4,1 & 1,4 & $\overline{0,7}$ & & 17,0 & $\overline{4,9}$ \\
\hline
\end{tabular}

${ }^{1}$ largeur maximale à l'attache des mandibules ; ${ }^{2}$ largeur sous les yeux ; ${ }^{3}$ largeur au milieu ; ${ }^{4}$ longueur de l'émargination. 
les localités où l'espèce est présente, une stridulation composée de phrases répétées similaires à celle illustrée figure 15 on pu être enregistrées (ces phrases sont légèrement plus longues et sont séparées par des pauses plus courtes). Il est possible qu'il s'agisse de la forme continue du chant d'appel de A. cesairei $\mathrm{n}$. sp. (ces deux types de chants sporadique/continu existent également chez Salomona redtenbacheri Brongniart, 1897, une autre Agraeciini [Hugel, in prep.]).

Etymologie. - Cette espèce martiniquaise est dédiée à Aimé Césaire.
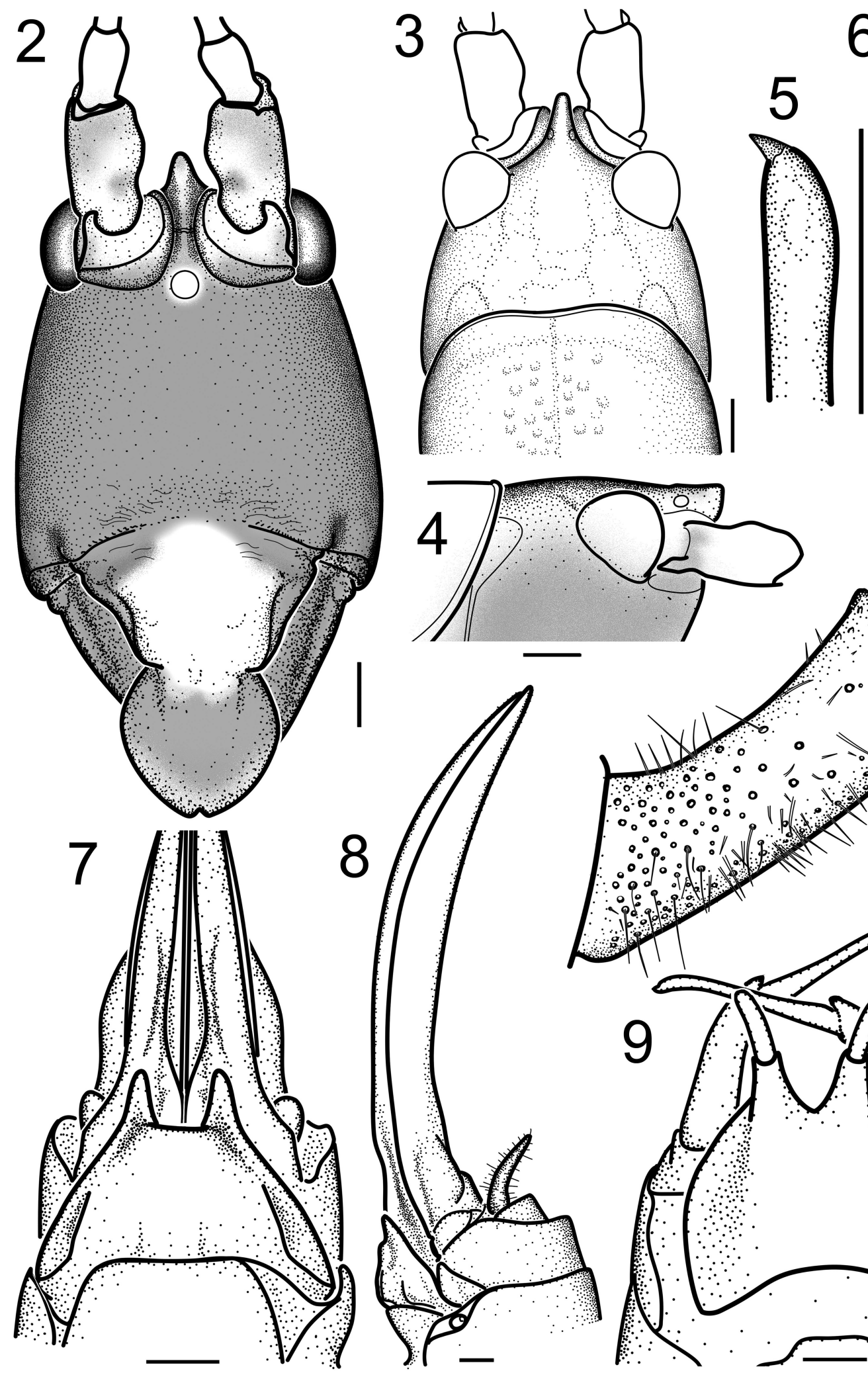

6
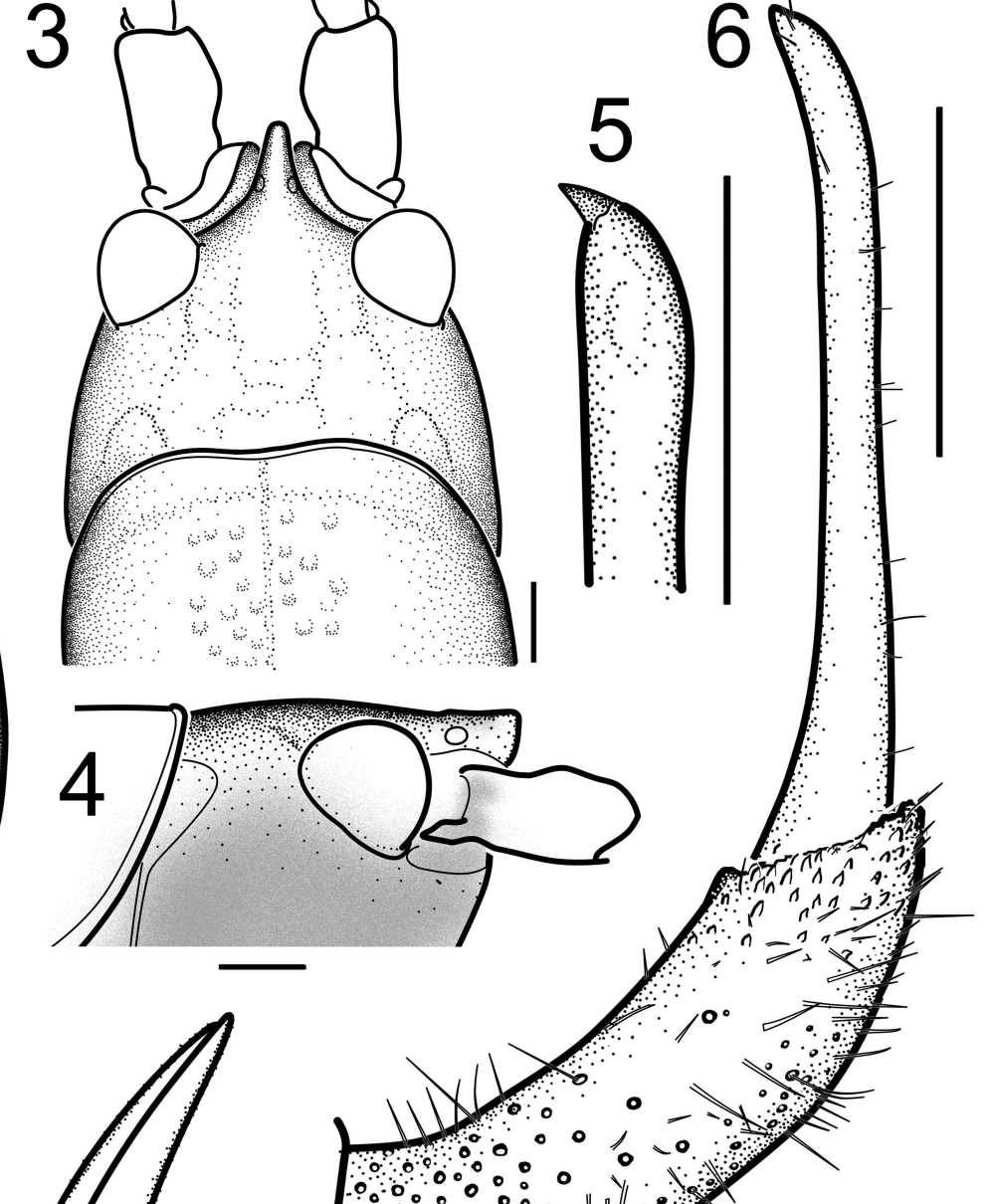

Fig. 2-9. - Agraecia cesairei n. sp. - 2-4, Tête du ô en vue frontale (2), dorsale (3) et latérale droite (4). - 5-6, Cerque gauche du $\widehat{o}$ en vue supérieure (6) et détail de l'apex en vue postérieure (5), haut à gauche, bas à droite. -7 , Plaque sous-génitale de la + en vue ventrale. -8 , Oviscapte en vue latérale droite. -9 , Plaque sous-génitale du ${ }^{\hat{A}}$ en vue ventrale. Echelles : $1 \mathrm{~mm}$. 

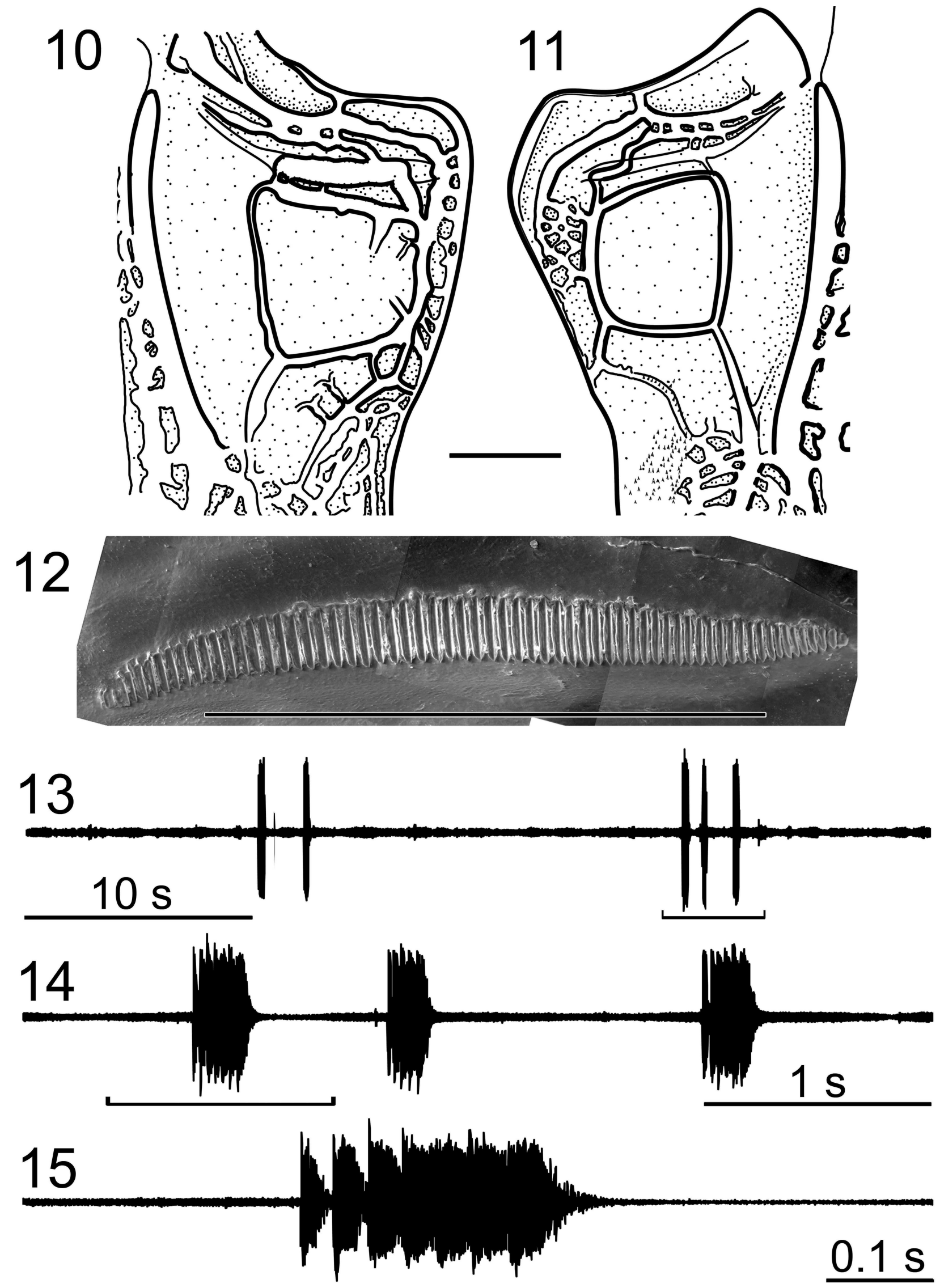

Fig. 10-15. - Agraecia cesairei n. sp., structures stridulatoires du đ et stridulation. - 10-11, Base de l'élytre gauche (10) et droit (11) en vue dorsale. - 12, Râpe stridulatoire (élytre gauche en vue ventrale). Image réalisée avec le microscope électronique à balayage de l'Institut Pluridisciplinaire Hubert Curien, DEPE, UMR 7168, Strasbourg, en mode environnemental, sans métallisation. - 13-15, Chant d'appel de l'holotype enregistré à $22 \mathrm{H} 00$, à $26^{\circ} \mathrm{C}$, in natura avec un détecteur d'ultrasons Pettersson D230 (hétérodyne sur $22 \mathrm{kHz}$ et division de fréquence par 10) sur un caméscope Sony HDR HC1E (échantillonnage 48kHz) et analysé avec Clampfit 9.2.1.8. Echelles : $1 \mathrm{~mm}$. 
Biologie. - Agraecia cesairei $\mathrm{n}$. sp. a été trouvé dans trois localités du massif du Carbet entre 500 et 700 mètres d'altitude, dans la série hygrophile, systématiquement en sous-bois dans des milieux ou la strate arbustive est bien développée. Cette sauterelle est uniquement active de nuit et a été observée sur différentes plantes de la strate arbustive (observée sur Pipéracées, Melastomatacées, Héliconiacées, Fougères). En captivité, A. cesairei n. sp. se nourrit de petits insectes, de feuilles et de fruits, les femelles pondent en hauteur dans des branches mortes, l'incubation des œufs dure une centaine de jours à $20^{\circ} \mathrm{C}$. Par ailleurs, une photographie de cette espèce est publiée dans le livre de SASTRE \& BREUIL $(2007$ : 213) sur la flore des Antilles françaises. Cette espèce occupe la même niche écologique (orthoptère nocturne omnivore à dominante insectivore, arboricole de la strate arbustive de la série hygrophile) et les mêmes fréquences dominantes que les Copiphorinae Acantheremus bonfilsi Hugel \& Morin, 2003, en Guadeloupe (Hugel \& Morin, 2003) et à Marie-Galante, et Acantheremus dominicanus Naskrecki, 1997, en Dominique et également à Marie-Galante (Hugel, obs. pers.).

\section{Agraecia viridipennis Redtenbacher, 1891}

Agraecia viridipennis Redtenbacher, 1891 : 453. Holotype (†) : Brasilien [Brésil], coll. Br[unner] v[on] W[attenwyl] ex. coll. Fischer (Naturhistorisches Museum Wien ; photographies à forte résolution non publiées examinées).

Agraecia malkini Piza, 1978 : 185, n. syn. Holotype ( $\left.{ }^{3}\right)$ : Brazil [Brésil], Igarapé Gurupi, Uma Aldeia Araçu, Ma; 50 km E. de Canindé, 20.V.1963, Malkin (Museu de Zoologia da Universidade de São Paulo ; photographies de EADES \& OTTE, 2009, examinées).

SPÉCIMENS EXAMINÉS : $\widehat{\jmath}$, Guyane française, Régina, piste de Kaw pk 36, ouest du camp Patawa, 175 m alt., 5209'12"O - 04³2'36"N, 9.X.2004, S. Hugel, coll. S. Hugel ; +, idem ; 2 \%, 18-25.VIII.2000, S. Hugel, coll. S. Hugel.

Trois femelles et un mâle de Agraecia appartenant manifestement à la même espèce ont été collectés dans la même localité de Guyane française. Les femelles correspondent en tout point à la description originale et à l'holotype femelle de $A$. viridipennis Redtenbacher, 1891, et le mâle correspond en tout point à la description originale très succincte (PIZA DE TOLEDO, 1978) et aux photos disponibles de l'holotype mâle de A. malkini Piza, 1978 (EADES \& OTTE, 2009). Je considère donc que :

Agraecia viridipennis Redtenbacher, 1891 = Agraecia malkini Piza, 1978, n. syn.

Diagnose. - Cette espèce se distingue de toutes les autres Agraecia néotropicales par la combinaison des caractères suivants: face vert pâle, clypéus et labre vert sombre, ocelles et apex du fastigium jaune-orange, aucune tache noire (bandes noires chez A. nigrifrons Redtenbacher, 1891, et A. ornata Karny, 1907, face noire chez A. cesairei n. sp.) ; face relativement large (étroite et allongée chez A. cesairei $\mathrm{n}$. sp.); ocelle médian de très grande taille, bien plus large qu'un œil, son centre situé très au-dessous du niveau de la base des scrobes antennaires (ocelle médian moins large qu'un œil chez A. incognita, A. nigrifrons, A. ornata, A. punctata); face lisse (avec des dépressions chez A. ornata); apex du fastigium du vertex arrondi (en éperon chez A. subulata Redtenbacher, 1891 ; en pointe chez A. maculata Redtenbacher, 1891); fastigium du vertex frontalement formant une crête étroite (large et aplati chez A. incognita Piza, 1970) ; longueur des élytres $>35 \mathrm{~mm}(<25 \mathrm{~mm}$ chez A. festae $)$ élytres dépassant largement les genoux postérieurs (les atteignant à peine chez A. abbreviata Redtenbacher, 1891 et $A$. vittipes Redtenbacher, 1891, et les dépassant légèrement chez A. ornata); base du champ costal des élytres avec dans les cellules des taches sombres irrégulières aux limites bien nettes ; fémurs antérieurs sans épines ventrales postérieures ; fémurs postérieurs élancés, plus de 6 fois aussi longs que large (fémurs postérieurs trapus, environ 4 à 4,5 fois plus longs que large chez A. vittata Redtenbacher, 1891, et A. pulchella Hebard, 1927); miroir de l'élytre 
gauche du mâle avec de nombreuses veines renflées dans la zone anale (miroir gauche translucide et membraneux chez A. dorsalis, A. festae, A. nigrifrons, A. ornata, A. punctata, A. subulata); plaque sous-génitale du mâle avec une émargination profonde (émargination superficielle chez $A$. incognita); cerques du mâle prolongés par une protrusion latérale dirigée vers le bas et l'arrière et une très longue protrusion postérieure s'incurvant vers le bas et l'intérieur et finissant en pointe (cerques du mâle sans très longue protrusion chez A. dorsalis Karny, 1907, A. punctata, A. subulata et A. vittipes) ; plaque sous-génitale de la femelle avec une émargination superficielle (émargination profonde et carrée chez A. cesairei $\mathrm{n}$. sp, profonde en $\mathrm{V}$ chez A. incognita, profonde chez A. pulchella); oviscapte égal à 80-90\% de la longueur du fémur postérieur (à peine plus court chez $A$. vittipes et $A$. ornata); oviscapte légèrement et régulièrement incurvé vers le haut (brusquement incurvé au milieu chez A. pulchella; excurvé chez A. incognita), longueur $>15 \mathrm{~mm}$ (9 mm chez A. festae).

Redescription. - Outre les caractéristiques générales du genre (KARNY, 1912).

Couleur. Adultes : coloration générale vert pâle, jaune sur les spécimens de collection ; face sans tache noire; clypéus, labre, mandibules vert plus sombre (jaune chez les spécimens vivants ?); ocelle médian, ocelles latéraux et apex du fastigium jaune-orange (plus ou moins décoloré chez les spécimens de collection); élytres vert pâle avec des taches noires bien nettes dans certaines cellules, particulièrement dans le champ costal.

Tête. Face lisse. Fastigium du vertex atteignant le milieu du scape (vue frontale, fig. 16), dépassant à peine les scrobes antennaires (vue dorsale, fig. 17); apex du fastigium arrondi (en vue latérale, fig. 18); base du fastigium du vertex étroite, concave, largement dépassée par les scrobes antennaires (vue semilatérale, scapes relevés). Ocelle médian bien distinct, très grand (fig. 16), ovale, diamètre très supérieur à celui d'un œil, centre situé très au-dessous du niveau de la base des scrobes, occupant environ le tiers de la largeur de la face. Occiput avec des sillons longitudinaux et transverses irréguliers. Yeux petits, peu saillants. Antennes $>80 \mathrm{~mm}$.

Thorax. Pronotum : brillant, avec de nombreuses dépressions et rides; bord antérieur droit ou très légèrement concave (fig. 17), bord postérieur légèrement convexe ; sillons antérieur et postérieur bien distincts sur le disque ; pas de sillon sagittal distinct ; bord ventral du lobe latéral non concave ; sinus huméral non concave. Epines du prosternum longues.

Elytres. Dépassant nettement les genoux postérieurs.

Pattes. Tibia antérieur : arrondi dorsalement, aplati ventralement, avec 1 éperon apical ventral de part et d'autre, et ventralement 5 éperons subapicaux internes et 5 éperons subapicaux externes ; renflement tympanique très élargi, environ 2 fois plus large que le tibia (vue frontale), orifices tympaniques largement ouverts vers l'avant, aussi larges que l'espace qui les sépare. Fémur antérieur : arrondi dorsalement, avec deux carènes longitudinales ventrales, avec 3-5 épines ventrales internes ( 3 chez l'holotype et 3-4 chez l'holotype de A. malkini), sans épines ventrales externes; lobe géniculaire interne finissant en épine ; lobe géniculaire externe pointant légèrement ventralement. Epine de la coxa antérieure large en vue dorsale. Tibia médian : arrondi dorsalement, aplati ventralement à l'apex ; avec un éperon apical ventral de part et d'autre ; avec ventralement 6-7 éperons subapicaux antérieurs (6 chez l'holotype) et 5-6 éperons subapicaux postérieurs (7 chez l'holotype). Fémur médian : arrondi dorsalement ; avec une carène ventrale antérieure et une carène ventrale postérieure ; avec 4-6 épines ventrales antérieures (4-5 chez l'holotype et 5 chez l'holotype de A. malkini); sans épines ventrales postérieures ; lobes géniculaires terminés par une épine de part et d'autre. Coxa médiane avec une petite épine distale dorsale antérieure et un tubercule distal ventral. Pattes postérieures absentes sur l'holotype. Tibia postérieur : arrondi ventralement, apex aplati (deux carènes ventrales apparaissent à l'apex); aplati dorsalement ; avec 2 éperons apicaux ventraux de part et d'autre et 1 éperon apical dorsal de part et d'autre ; avec 6-7 éperons subapicaux ventraux internes; avec 8-10 éperons subapicaux ventraux externes; avec 13 épines dorsales internes, et 11-12 épines dorsales externes. Fémur postérieur avec deux carènes ventrales ; carène ventrale interne avec 1-2 épines ; carène ventrale externe avec 7-8 épines (A. malkini holotype : 8) ; lobes géniculaires terminés par une longue épine effilée de part et d'autre.

Dimorphisme sexuel. Hormis les caractères sexuels primaires, les deux sexes sont similaires; les mâles sont très légèrement plus petits que les femelles. 

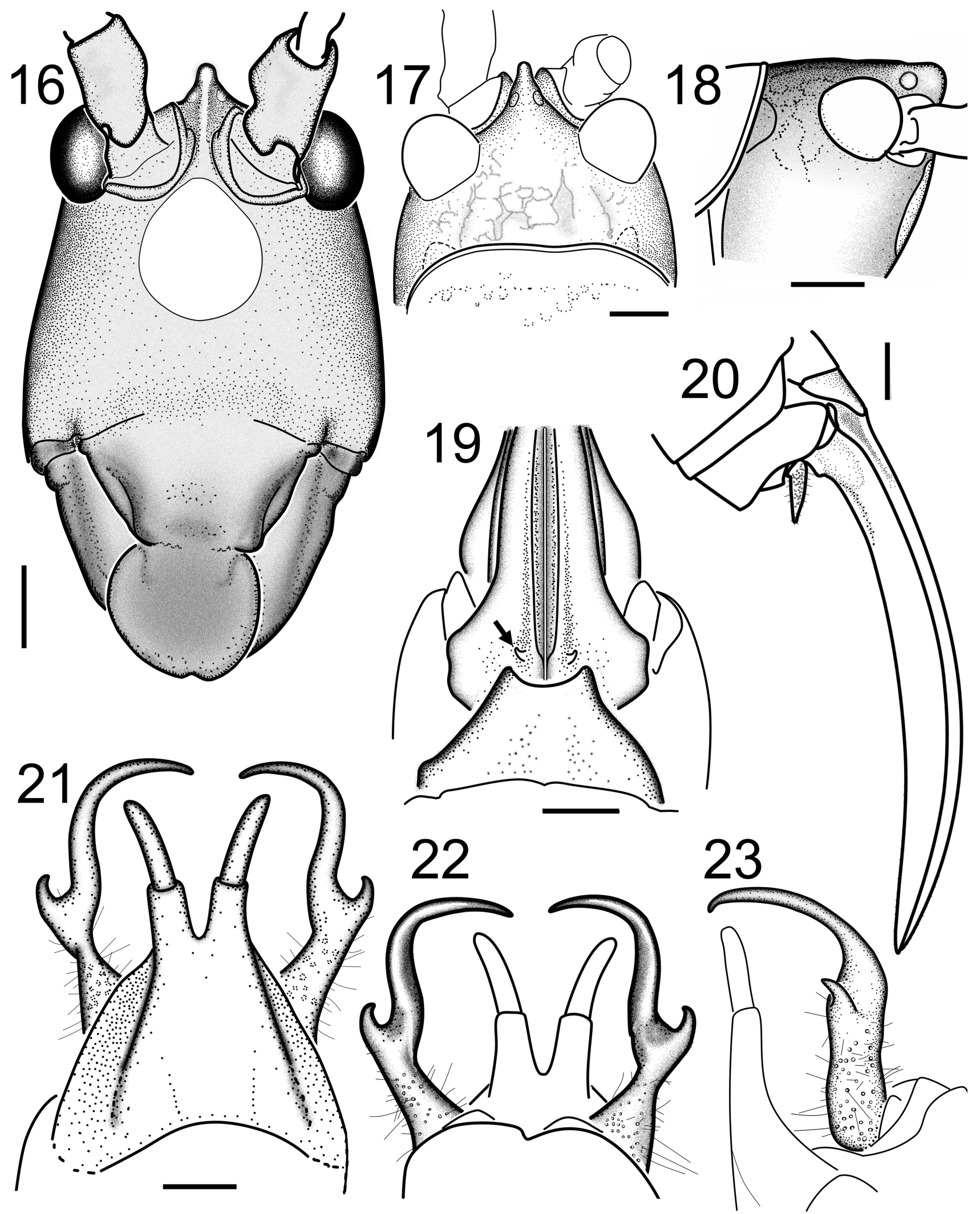

Fig. 16-23. - Agraecia viridipennis Redtenbacher, 1891. - 16-18, Tête du đ̂ en vue frontale (16), dorsale (17) et latérale droite (18). - 19, Plaque sous-génitale de la $q$ en vue ventrale. - 20, Oviscapte en vue latérale droite. - 21-23, Plaque sous-génitale et cerques du $\widehat{o}$ en vue ventrale (21) dorsale (22) et latérale droite. Echelles : $1 \mathrm{~mm}$.

Mâle. - Appareil stridulatoire. Elytre gauche (fig. 24 et 26) : crête stridulatoire en vue dorsale de $1.8 \mathrm{~mm}$ environ (vue dorsale); râpe avec 124 lames (fig. 26) ; miroir non transparent, avec de nombreuses veines, principalement dans le cadran antéro-médian (élytre fermé). Elytre droit (fig. 25) : miroir au milieu plus long que large (élytre fermé ; environ 1,4 fois plus long au milieu que large au milieu); distalement au miroir, champ anal avec de nombreuses soies courtes spiniformes. Aile. Base de l'aile sans évagination molle. Terminalia. Plaque suranale avec une petite échancrure (mal préservé chez l'holotype de A. malkini et le spécimen de Guyane) ; cerques (fig. 21, 22 et 23) très allongés, base 
large avec de nombreuses soies; base prolongée d'une courte épine latérale dirigée vers l'arrière et le bas, et d'une longue protrusion postérieure s'incurvant vers le bas et l'intérieur et finissant en pointe ; base de la protrusion (au niveau de l'épine latérale) aplatie latéralement, tiers apical de la protrusion cylindrique. Plaque sous-génitale (fig. 21) allongée, environ aussi longue (sans les styli) que large à la base ; bord postérieur avec une échancrure étroite en $\mathrm{V}, 0,2$ fois aussi profonde que la longueur maximale de la plaque sous-génitale ; carènes latérales distinctes distalement, convergentes ; styli bien distincts, plus longs que la profondeur de l'émargination. Genitalia. Titillateurs (fig. 28) symétriques, courts, plus larges que hauts, accolés au milieu, bien sclérifiés, apex avec des denticules dirigés vers le haut.
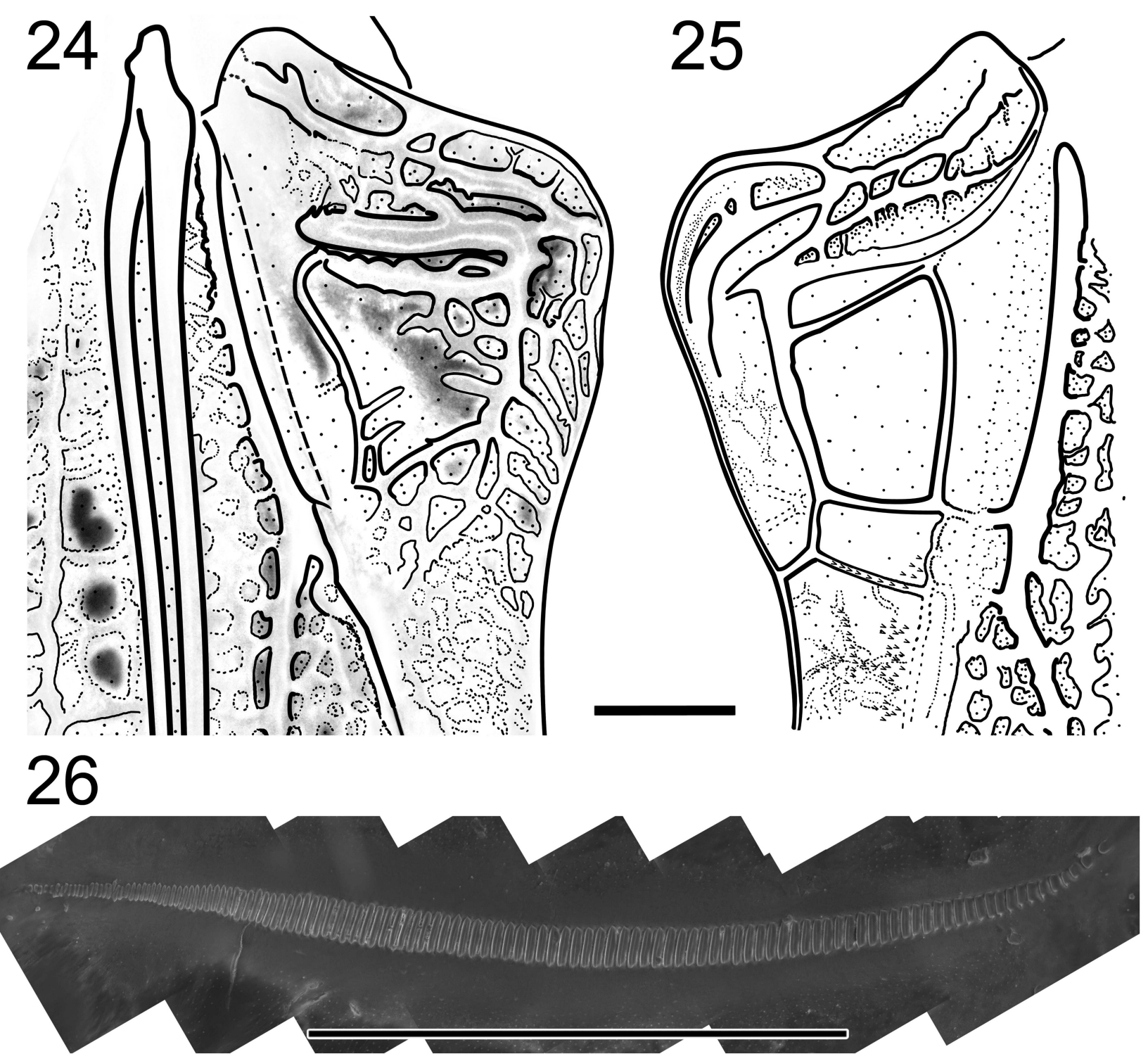

Fig 24-26. - Agraecia viridipennis Redtenbacher, 1891, structures stridulatoires du ô. - 24-25, Base de l'élytre gauche (24) et droit (25) en vue dorsale. - 26, Râpe stridulatoire (élytre gauche en vue ventrale). Image réalisée avec le microscope électronique à balayage de l'Institut Pluridisciplinaire Hubert Curien, DEPE, UMR 7168, Strasbourg, en mode environnemental, sans métallisation. Echelles : $1 \mathrm{~mm}$.

Femelle. - Terminalia : sternite précédant la plaque sous-génitale avec un petit tubercule au milieu ; plaque sous-génitale (fig. 19 et 20) plus large que longue, sans carènes bien distinctes ; émargination apicale très superficielle, arrondie ; oviscapte régulièrement incurvé dès la base, avec de minuscules denticulations peu distinctes sur les valves dorsales à partir du milieu, et à l'apex des valves ventrales ; base des valves ventrales avec deux petites épines dirigées vers l'extérieur (fig. 19, flèche).

Biologie. - A. viridipennis a été observé en sous-bois, de nuit, entre 1 et 3 mètres de haut. Le site était très fermé lors des collectes (non exploité et sans chablis). Il est localisé en bordure d'une zone marécageuse. 
Tableau II. - Mesures de Agraecia viridipennis Redtenbacher, 1891 (en mm).

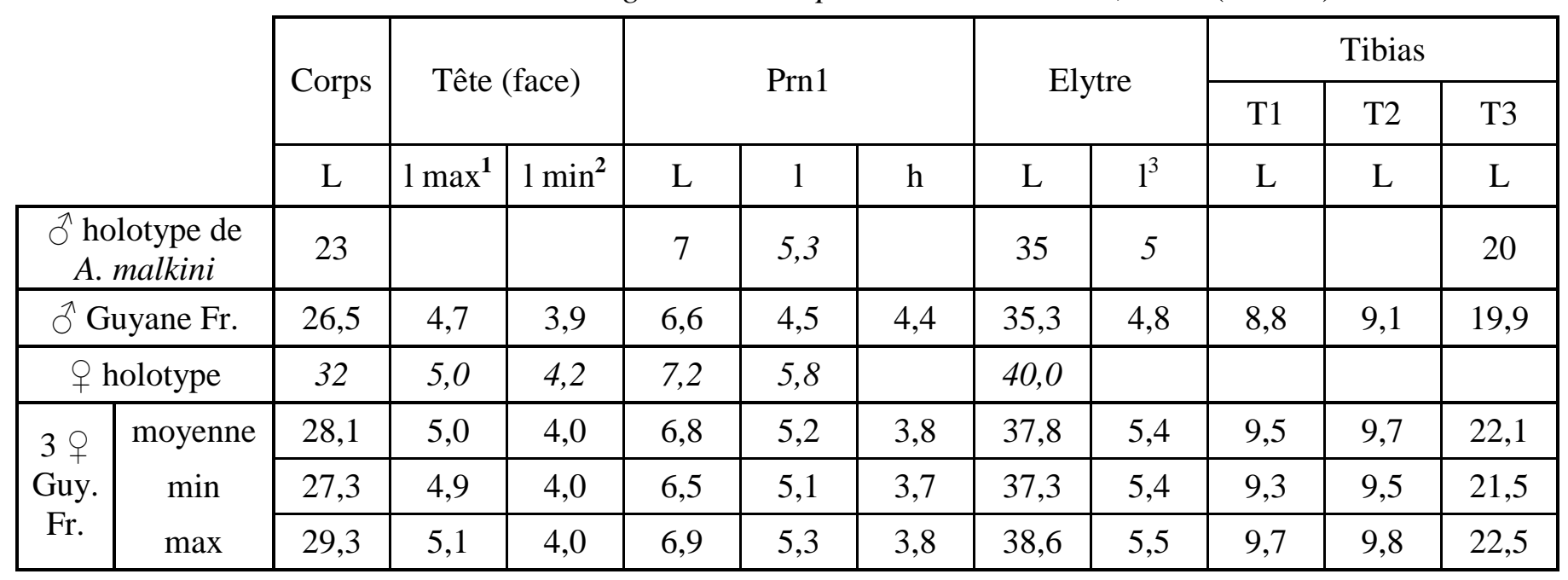

\begin{tabular}{|c|c|c|c|c|c|c|c|c|c|c|c|c|}
\hline & \multicolumn{4}{|c|}{ Fémurs } & \multirow{2}{*}{\multicolumn{4}{|c|}{ Plaque sous-génitale }} & \multirow{3}{*}{$\begin{array}{c}\text { Styli } \\
\mathrm{L}\end{array}$} & \multirow{2}{*}{\multicolumn{2}{|c|}{ Oviscapte }} \\
\hline & & \multirow{2}{*}{$\frac{\mathrm{F} 1}{\mathrm{~L}}$} & \multirow{2}{*}{$\frac{\mathrm{F} 2}{\mathrm{~L}}$} & \multicolumn{2}{|c|}{ F3 } & & & & & & & \\
\hline & & & & $\mathrm{L}$ & $1 \max$ & L max & 1 base & 1 apex & Lém ${ }^{4}$ & & $\mathrm{~L}$ & $1^{3}$ \\
\hline \multicolumn{2}{|c|}{$\begin{array}{c}\text { रे holotype de } \\
\text { A. malkini }\end{array}$} & 8 & & 18 & & & & & & & & \\
\hline \multicolumn{2}{|c|}{${ }^{\lambda}$ Guyane Fr. } & 8,1 & 8,2 & 19,4 & 3,1 & 4,0 & 4,0 & 1,4 & 0,8 & 1,3 & & \\
\hline \multicolumn{2}{|c|}{ o holotype } & & & & & 2,0 & 4,0 & 1,2 & 0,2 & & 18,0 & 2,0 \\
\hline \multirow{3}{*}{$\begin{array}{c}3 \text { q } \\
\text { Guy. } \\
\text { Fr. }\end{array}$} & \multirow{3}{*}{$\begin{array}{c}\text { moyenne } \\
\min \\
\max \end{array}$} & 8,6 & 8,8 & 20,6 & 3,4 & 1,7 & 3,0 & 1,0 & 0,3 & & 17,2 & 1,9 \\
\hline & & 8,5 & 8,7 & 20,0 & 3,3 & 1,6 & 2,6 & 0,9 & 0,2 & & 16,2 & 1,9 \\
\hline & & 8,7 & 8,9 & 21,0 & 3,5 & 1,9 & 3,4 & 1,2 & 0,4 & & 17,8 & 2,0 \\
\hline
\end{tabular}

${ }^{1}$ largeur maximale à l'attache des mandibules $;{ }^{2}$ largeur sous les yeux $;{ }^{3}$ largeur au milieu ; ${ }^{4}$ longueur de l'émargination. Les valeurs en italique sont interpolées d'après des photographies.

\section{DISCUSSION}

Le genre Agraecia qui inclut actuellement 17 espèces mériterait une révision. Parmi les 17 espèces considérées comme appartenant à ce genre, les espèces orientales et africaines devraient probablement être rattachées à d'autres genres (existants ou nouveaux). Par ailleurs, A. vittata Redtenbacher, 1891, décrite d'après un spécimen femelle subadulte, et $A$. pulchella Hebard, 1927, décrite d'après une femelle adulte, sont très probablement synonymes. Les types proviennent tous deux de Colombie, se distinguent de tous les autres Agraecia néotropicaux par les fémurs postérieurs très trapus, et se distinguent uniquement l'un de l'autre par une coloration différente du pronotum (HEBARD, 1927). Le cas de A. cesairei $\mathrm{n}$. sp. indique que de telles différences de coloration entre un spécimen jeune et un adulte existent au sein d'une même espèce d'Agraecia (voir plus haut) et ne peuvent donc pas être considérés comme des caractères diagnostiques pour ce genre. Enfin, alors que certaines espèces néotropicales forment clairement un groupe homogène avec notamment un fastigium comparable (notamment $A$. abbreviata, A. cesairei n. sp., A. nigrifrons, A. ornata, A. pulchella, A. punctata, A. viridipennis, A. vittata, $A$. vittipes), d'autres espèces devraient être rattachées à des genres distincts $(A$. incognita et peut-être A. maculata et A. subulata).

REMERCIEMENTS. - Je remercie le Parc naturel régional de la Martinique et principalement Mesdames B. Chanteur et L. N'Guela pour m'avoir autorisé à collecter des échantillons dans le Parc. Je remercie également Jonathan Weber et Marilyne Boucherit pour avoir recherché et collecté deux spécimens de A. cesairei n. sp. lors de leurs vacances, David Casimirius pour avoir recherché des spécimens supplémentaires, Christian Bouladou 
Dupré pour m'avoir fait part de ses observations, la famille Gensse pour leur aide logistique, Franziska Anderle (Naturhistorisches Museum Wien) pour les photographies de l'holotype de A. viridipennis. Je remercie mes collègues et amis entomologistes de Guyane et particulièrement Odette Morvan et Jean-Aimé Cerda pour leur accueil et leur aide, et Roland Lupoli, Jean-Philippe Champennois, Nicolas Heck et mon père pour m'avoir accompagné sur le terrain. Merci à Jean-Hervé Lignot de l'Institut Pluridisciplinaire Hubert-Curien, DEPE, UMR 7168, Strasbourg, pour l'accès au MEB.

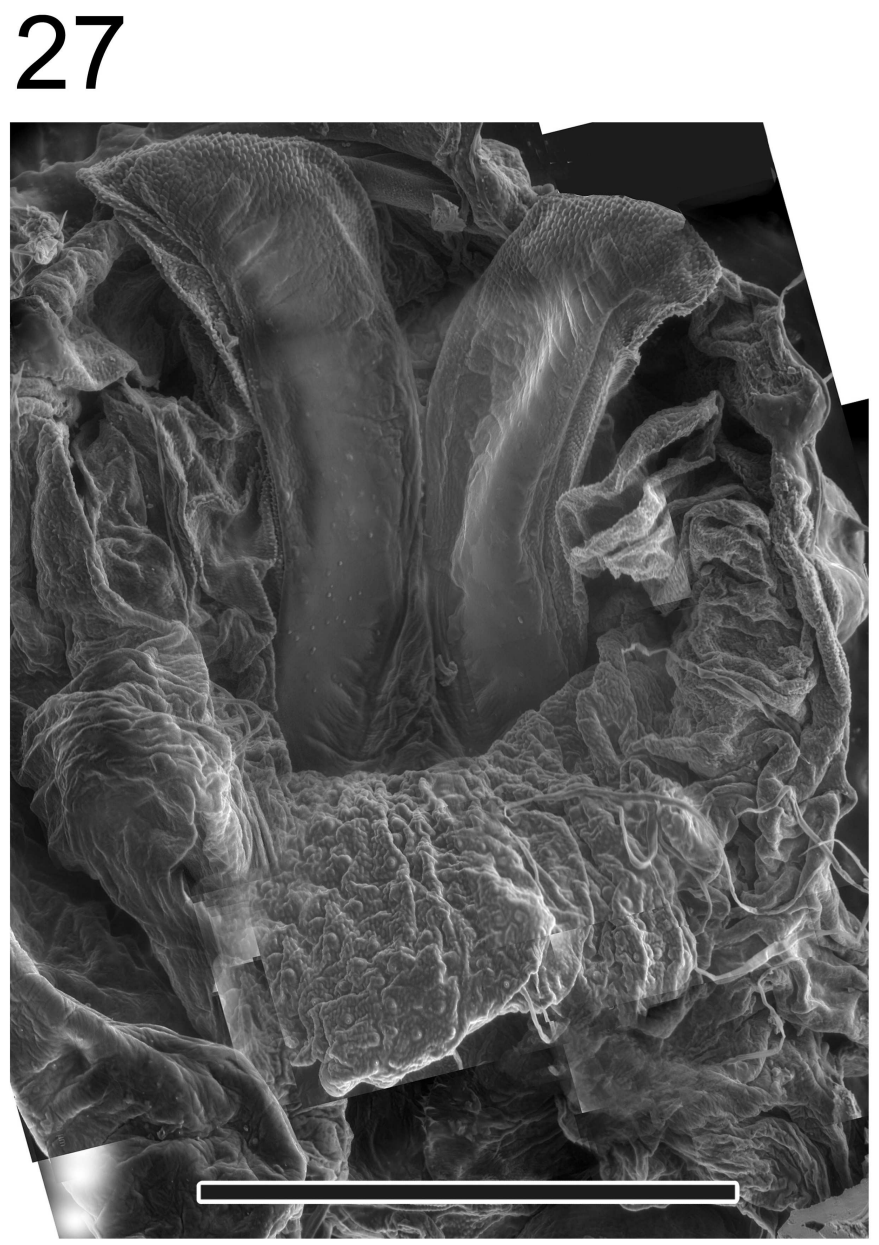

\section{8}

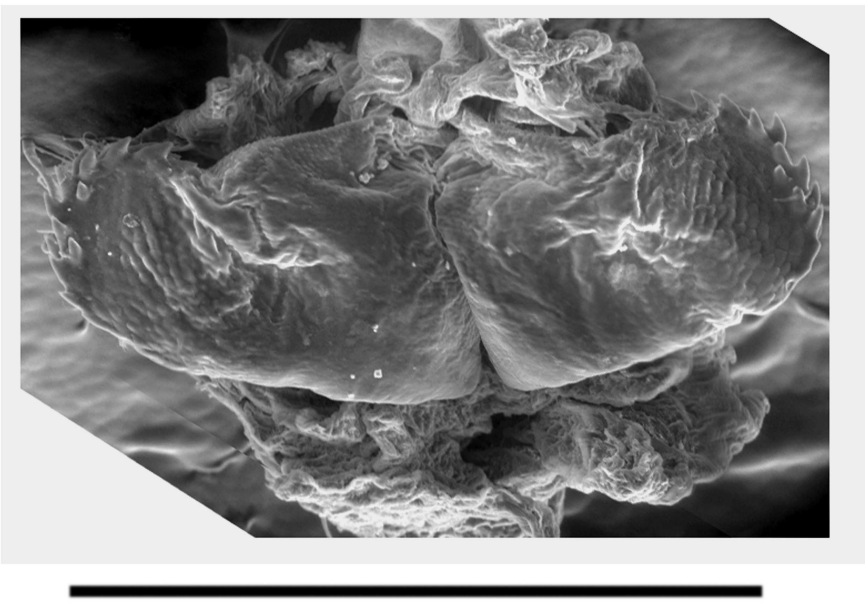

Fig 27-28. - Agraecia, genitalia : titillateurs en vue postérieure. Images réalisées avec le microscope électronique à balayage de l'Institut Pluridisciplinaire Hubert Curien, DEPE, UMR 7168, Strasbourg, en mode environnemental, sans métallisation. 27, Agraecia cesairei n. sp. - 28, A. viridipennis Redtenbacher, 1891. Echelles : $1 \mathrm{~mm}$.

\section{AUTEURS CITÉS}

Audinet-Serville J. G., 1831. - Revue méthodique des insectes de l'Ordre des Orthoptères. Annales des Sciences Naturelles Zoologie et Biologie Animale, 22 : 28-65.

EAdes D. C. \& OTTE D., 2009. - Orthoptera Species File Online. Version 2.0/3.5. [10.II.2009]. http://Orthoptera.SpeciesFile.org.

HeBARD M., 1927. - Studies on the Dermaptera and Orthoptera of Colombia. Fourth Paper, orthopterous Family Tettigoniidae. Transactions of the American Entomological Society, 52 [1926] : 275-354.

HugEL S. \& MORIN D., 2003. - Une espèce nouvelle d'Acantheremus de Guadeloupe (Orthoptera : Tettigoniidae : Copiphorinae). Bulletin de la Société entomologique de France, 108 (4) : 427-432.

KARnY H. H., 1912. - Orthoptera Fam. Locustidae Subfam. Agraeciinae. In : Wytsman, P. Genera Insectorum, 141 : 1-47.

PIZA S. DE TOLEDO, 1978. - Uma Agraeciinae e Uma Pseudophyllinae Novas Do Brasil (OrthopteraTettigoniidae). Revista de Agricultura (Piracicaba), 53 (4) : 185-187.

SASTRE C. \& BreuIL A., 2007. - Plantes, milieux et paysages des Antilles françaises. Collection Parthénope, Editions Biotope, Mèze, France. 672 p. 